\title{
Can Disordered Mobile Phone Use Be Considered a Behavioral Addiction? An Update on Current Evidence and a Comprehensive Model for Future Research
}

\author{
Joël Billieux ${ }^{1,2} \cdot$ Pierre Maurage $^{1,2} \cdot$ Olatz Lopez-Fernandez ${ }^{1,2}$. \\ Daria J. Kuss ${ }^{3} \cdot$ Mark D. Griffiths $^{3}$
}

Published online: 9 April 2015

(C) Springer International Publishing AG 2015

\begin{abstract}
Despite the many positive outcomes, excessive mobile phone use is now often associated with potentially harmful and/or disturbing behaviors (e.g., symptoms of deregulated use, negative impact on various aspects of daily life such as relationship problems, and work intrusion). Problematic mobile phone use (PMPU) has generally been considered as a behavioral addiction that shares many features with more established drug addictions. In light of the most recent data, the current paper reviews the validity of the behavioral addiction model when applied to PMPU. On the whole, it is argued that the evidence supporting PMPU as an addictive behavior is scarce. In particular, it lacks studies that definitively show behavioral and neurobiological similarities between mobile phone addiction and other types of legitimate addictive behaviors. Given this context, an integrative pathway model is proposed that aims to provide a theoretical framework to guide future research in the field of PMPU. This model highlights that PMPU is a heterogeneous and multi-faceted condition.
\end{abstract}

Keywords Behavioral addiction - Mobile phone addiction · Cyber addiction $\cdot$ Mobile phone problematic use

This article is part of the Topical Collection on Technology and Addiction

Joël Billieux

Joel.Billieux@uclouvain.be

1 Laboratory for Experimental Psychopathology, Psychological Sciences Research Institute, Université catholique de Louvain, 10, Place du Cardinal Mercier, 1348 Louvain-La-Neuve, Belgium

2 Internet and Gambling Disorders Clinic, Department of Adult Psychiatry, Cliniques Universitaires Saint-Luc, Brussels, Belgium

3 International Gaming Research Unit, Nottingham Trent University, Nottingham, UK

\section{Introduction}

Mobile phone use has substantially increased over the last decade, particularly in European and Asian countries. For instance, in Asia, the Office of the Communication Authority of Hong Kong (2013) stated that in 2012, the average number of cellular phones owned was 2.21. Regarding Europe, recent statistics by the Swiss Federal Statistics Office (2014) indicated that in 2012, there were more mobile phone subscriptions than inhabitants in many European countries (e.g., Finland, 1.72; Austria, 1.61; Italy, 1.60; UK, 1.35; Switzerland, 1.30; Sweden, 1.25; Germany, 1.12; and Belgium, 1.11).

Linked with this blooming of mobile phone use, research has recently flourished to explore both positive and negative consequences associated with mobile phone use. Positive outcomes of mobile phone use include the optimization of communication between individuals and systems [1]. Mobile phone technology also promoted the development and validation of a wide range of health-related and behavior change interventions and applications that aid such activities as dietary management, smoking cessation, physical activity promotion, and chronic disease management $[2,3]$. Negative outcomes associated with mobile phone use-particularly excessive use- have included (but are not restricted to) selfreported dependence and addiction-like symptoms [4, 5, 6••], sleep interference [7], financial problems [4], dangerous use (phoning while driving) [4, 8, 9], prohibited use (phoning in banned areas) $[4,10]$, and mobile phone-based aggressive behaviors (e.g., cyber bullying) [11].

Despite accumulating evidence that mobile phone use can become problematic and lead to negative consequences, its precise incidence, prevalence, and symptomatology remain a matter of much debate. As an illustration, prevalence studies conducted within the last decade 
have reported highly heterogeneous rates of problematic use ranging from just above $0 \%$ to more than $35 \%$ [12-15]. As discussed in more detail later in the paper, this heterogeneity is mainly due to the fact that most studies in the field have been conducted in the absence of a theoretical rationale. The prototypical example is that too often, excessive mobile phone use is conceptualized as a behavioral addiction and subsequently developed screening tools are adapted from the substance use and pathological gambling literature, without taking into account either the specificities of mobile phone "addiction" (e.g., dysfunctional mobile phone use may often be related to interpersonal processes) or the fact that the most recent generation of mobile phones (i.e., smartphones) are tools that - like the Internet - allow the involvement in a wide range of activities going far beyond traditional oral and written (SMS) communication between individuals (e.g., gaming, gambling, social networking, shopping, etc.). For example, it has recently been found that the involvement in social networking promotes the development of dependence toward mobile phone use [16]. As a consequence, many existing studies were based on an excessive simplification of heterogeneous and multi-faceted problematic behaviors, taking place between an individual and a technological instrument with constantly evolving applications.

Recently - and notwithstanding inconsistencies in prevalence rates reported in previous studies and the limited evidence regarding its etiology - problematic mobile phone use (PMPU) has raised sufficient concerns for being considered as a potential public health issue. For instance, in August 2014, the World Health Organization (WHO) hosted a meeting (in Tokyo, Japan) to discuss the public health implications and excessive behaviors associated with the use of information and communication technology, including mobile phones and smartphones. ${ }^{1}$ In this context, the objective of the current paper is twofold. First, the validity of the behavioral addiction model, when applied to PMPU, is discussed in light of the existing evidence. Second, an integrative pathway model that aims to provide a theoretical framework for future research on PMPU is proposed.

\section{Problematic Mobile Phone Use as a Behavioral Addiction}

The first empirical studies examining PMPU were published a decade ago by Australian and Japanese scholars [6••, 17]. Since then, the number of published studies on the topic has grown substantially [18], although mobile phone-related disorders have received less attention in the psychiatric and

\footnotetext{
${ }^{1}$ The first and the last authors of this paper were among the experts invited to attend the WHO meeting.
}

psychological literature than Internet-related disorders. Currently, several terms are frequently used to describe the phenomenon, the more popular being "mobile phone (or smartphone) addiction" [19], "mobile phone (or smartphone) dependence" [4], or "nomophobia" (that literally refers to the fear of not being able to use the mobile phone) [20]. Existing studies on the topic are mainly epidemiological and have attempted to estimate the prevalence of symptomatology of the condition and to delineate its psychosocial risk factors and comorbid psychopathology $[21 \bullet \cdot]$.

PMPU is generally conceptualized as a behavioral addiction including the core components of addictive behaviors, such as cognitive salience, loss of control, mood modification, tolerance, withdrawal, conflict, and relapse [15, 22, 23]. Accordingly, the criteria (and screening tools developed using such criteria) that have been proposed to diagnose an addiction to the mobile phone were directly transposed from those classifying and diagnosing other addictive behaviors, i.e., the criteria for substance use and pathological gambling depicted in the fourth revised edition of the Diagnostic and Statistical Manual of Mental Disorders (DSM-IV-TR) in the case of the first published studies on PMPU $[6 \bullet \bullet, 17]$. A decade on, scholars still generally tackle the issue of diagnosing PMPU using a similar approach. For example, in a recent study, Smetaniuk [13] reported a prevalence of PMPU around $20 \%$ in US undergraduate students using adapted survey items that were initially developed to diagnose disordered gambling.

Although many scholars believe that PMPU is a behavioral addiction, evidence is still lacking that either confirms or rejects such conceptualization. Indeed, the fact that this condition can be considered as an addiction is to date only supported by exploratory studies relying on self-report data which is collected via convenience samples. More specifically, there is a crucial lack of evidence that similar neurobiological and psychological mechanisms are involved in the etiology of mobile phone addiction compared to other chemical and behavioral addictions. Such types of evidence played a major role in the recent recognition of gambling disorder and Internet gaming disorder as addictive disorders in the latest edition of the DSM (i.e., DSM-5) [24, 25]. In particular, three key features of addictive behaviors, namely loss of control, tolerance, and withdrawal, have - to date-received very limited empirical support in the field of mobile phone addiction research.

First, several scholars proposed that loss of control (e.g., diminished impulse control and altered decision-making processes) is central in PMPU [21••]. Such a proposal was based on the extensive literature supporting that impulsivity is a hallmark of addictive behaviors [26, 27], including behavioral addictions such as disordered gambling [28, 29] and Internet addiction [30, 31]. Unfortunately, in the framework of PMPU, available evidence, although promising, has relied on either 
self-reports or clinical case studies. For example, it has been shown that questionnaires assessing emotion-based impulsivity and non-planning impulsivity correlate with measures of self-reported dependence to mobile phone and addictive pattern of use, as well as with negative outcomes such as financial problems $[4,5,32,33]$. A few studies have also described case reports of patients losing control over their use of the mobile phone $[20,34]$. However, there is a lack of empirical studies that have reported the behavioral and/or neurobiological indexes of impulsivity in mobile phone users. Further studies should therefore be conducted using recognized behavioral measures of inhibitory control (e.g., a stopsignal paradigm) and decision-making (e.g., Iowa gambling task). Ultimately, such types of impairment should be investigated and confirmed at the neurobiological level (e.g., using EEG or fMRI), similarly to studies on disordered gambling and Internet gaming disorder [30, 35, 36].

Second, relying on substance abuse criteria, attempts have been made to conceptualize the tolerance phenomenon with respect to PMPU. This has resulted in somehow weak conceptual formulations of what mobile phone tolerance comprises. For example, it has been proposed that in the framework of mobile phone addiction, tolerance is reflected by "a marked increase in the frequency and duration of mobile phone use to obtain satisfaction" or "the need to substitute operative devices with the new models that appear on the market" $[15,37,38]$. Although quite trivial, these operational definitions reflect the degree of conceptualization currently reached with regard to the tolerance construct applied to PMPU. In fact, within the framework of mobile phone use, inferring tolerance based on the increasing use of the mobile phone is highly tentative. Indeed, usage patterns can vary as a function of numerous contextual and social variables, including age (e.g., teenagers are more strongly influenced by peers), type of subscription (prepaid or subscriptions), relationship status (single or in couple), occupation (active versus employed, holidays versus working periods), and significant life events (e.g., beginning or end of a romantic relationship).

As for loss of control, the field lacks studies that have addressed the question in a more direct and valid way. If tolerance can be (at least partly) reflected by an increase in the actual use of the mobile phone, it is necessary to emphasize this by longitudinal designs rather than crosssectional self-report studies. Ultimately, it is not possible to assert any definitive conclusions regarding the existence of tolerance in individuals with PMPU unless neurobiological evidence (e.g., alteration/sensitization in specific cerebral circuitries) confirms it.

Third, tentative data interpretation has resulted in propositions that PMPU can be characterized by withdrawal, as found in substance addictions. However, the supporting evidence is scarce. Indeed, existing data comprises studies conducted using community samples (often students) emphasizing that some individuals claim via self-report to be upset when they are not able to use their mobile phone (e.g., when they have left it at home, when no network is available, when they have to switch if off) $[6 \bullet \bullet, 32,39,40]$. In such studies, typical items used for assessing withdrawal include "I feel restless and irritable when the mobile phone (or smartphone) is unavailable" or "I panic when I cannot use my mobile phone (or smartphone)." Unfortunately, these studies provide - at best — only indirect evidence of withdrawal. This is because elevated levels of self-reported distress when unable to use the mobile phone are susceptible to be triggered by a wide range of individual and contextual factors. For example, King et al. [20, 41] described cases of social phobia and panic disorder patients who displayed increased anxiety and discomfort when they could not use their mobile phone. Within the framework of panic disorder, the mobile phone can be used as an instrument that guarantees safety (i.e., it allows the calling of emergency services at any time), whereas in the framework of social phobia, the mobile phone allows the avoidance of direct social relations. In a previous paper, some of the present authors described the case of a woman with poor self-esteem and insecure attachment who became upset when she found herself in situations in which she could not use the mobile phone to call or send an SMS to her boyfriend [34]. In fact, to be able to assert that items such as those aforementioned actually measure withdrawal, it will be necessary to confirm their validity and specificity in longitudinal and/or experimental studies comparing people presenting with or without PMPU in situations of deprivation (i.e., assessing psychological and/or physiological symptoms in individuals after they have experienced a certain amount of time without having access to their mobile phone).

Given these concerns, it appears that the empirical evidence supporting the conceptualization of PMPU as a genuine addictive behavior is currently scarce. However, this does not necessary mean that PMPU is not a genuine addictive behavior (at least for a subgroup of individuals displaying PMPU symptoms), but rather that the nature and amount of the available data at the present time are not sufficient to draw definitive and valid conclusions. Therefore, further studies are required. In particular, longitudinal and experimental research is needed to obtain behavioral and neurobiological correlates of PMPU. In the absence of such types of data, all attempts to consider PMPU within the framework of behavioral addictions will remain tentative. It is worth noting here that it took decades of empirical research before disordered gambling was officially recognized as an addiction (as opposed to a disorder of impulse control) in the DSM-5.

The current conceptual chaos surrounding PMPU research can also be related to the fact that while the number of empirical studies is growing quickly, these studies have (to date) primarily been based on concepts borrowed from 
other disorders (e.g., problematic Internet use, pathological gambling, substance abuse, etc.). This approach is atheoretical and lacks specificity with regard to the phenomenon under investigation. In fact, by adopting such a "confirmatory approach" relying on deductive quantitative studies, important findings that are unique to the experience of PMPU have been neglected. As an illustration, no qualitative analyses of PMPU exist, and only a few models have been proposed. This implies that most studies have been conducted without a theoretical rationale that goes beyond transposing what is known about addictions in the analysis of PMPU [21••]. In such a context, our objective in the last section of this paper is to propose a comprehensive framework that aims to inspire and stimulate future research into PMPU. To do so, a model stipulating that different pathways can lead to PMPU is described. In this model, PMPU comprises, but is not limited to, addictive patterns of use.

\section{Toward a Pathway Model of Problematic Mobile Phone Use}

Research into PMPU is limited by the absence of a theoretical framework that allows for an understanding of its etiology and course. As described in this paper, existing studies have considered PMPU as a behavioral addiction a priori, without taking into account its uniqueness, as well as the heterogeneity of both its manifestations (e.g., addiction symptoms, banned use, risky use, etc.) and related risk factors (e.g., personality traits, comorbid psychopathology).

As depicted in Fig. 1, based on the available data from the literature, ${ }^{2}$ we hypothesized that at least three pathways can lead to PMPU: (1) an excessive reassurance pathway; (2) an impulsive-antisocial pathway; and (3) an extraversion pathway. Each of these pathways is influenced by individual factors (e.g., psychological characteristics of individuals) and can lead to various uses and misuses (e.g., frequency and type of use, risky use, addiction-like symptoms). Drawing on the available literature, the following sections provide the evidence supporting the existence of each of these pathways. It is worth noting that a preliminary attempt was proposed in 2012 to distinguish theoretical pathways leading to PMPU [21••]. However, since then, some of the authors' initial proposals have been refined and updated based on the latest available empirical data. Importantly, there is now a substantive amount of data that supports the relevance of the postulated pathways.

The first pathway leading to PMPU is the excessive reassurance pathway. This pathway corresponds to individuals

\footnotetext{
2 These three pathways have been proposed based on current evidence. It is however possible that future research will lead to considering other potential pathways to PMPU.
}

whose PMPU is driven by the necessity to maintain relationships and obtain reassurance from others. The objects of reassurance can be multiple. Some individuals use the mobile phone excessively to obtain reassurance in affective relationships [34]. As an illustration, recent data have shown that excessive and uncontrolled SMS use is related to concerns about relationship maintenance [42, 43•]. Moreover, using a cluster analytic approach, Lu et al. [43•] highlighted the existence of a subgroup of problematic users characterized by a poor self-model of adult attachment, along with increased anxiety and depression. These individuals, who display elevated actual and problematic use of mobile phones, were classified as "dependent users" by the authors. Other research also found that an increased level of general anxiety, as well as social anxiety, is associated with excessive mobile phone use and proneness to send a large number of SMSs [4, 14, 44, 45]. Such types of association have also been found in clinical case studies of panic disorder and socially phobic patients [20, 41]. Converging data also emphasize that high levels of neuroticism (i.e., the tendency to be emotionally vulnerable) [6••, 46-49] and low levels of self-esteem [6••, 14, 46, 48, 50] are associated with increased PMPU. On the whole, it therefore appears that addiction-like symptoms can be the consequence of a need for reassurance promoted by factors such as increased anxiety, poor self-esteem, insecure attachment, or increased emotional instability.

The second pathway leading to PMPU is the impulsive pathway. This pathway corresponds to individuals whose PMPU is driven by poor impulse control resulting in uncontrolled urges and deregulated use. Crucially, the impulsive pathway can lead to various manifestations of PMPU, namely addictive, antisocial, and/or risky patterns of use. For example, several research studies have shown that addictive patterns of use are associated with specific impulsivity traits, such as urgency (the tendency to act rashly in emotional contexts), lack of planning/premeditation (the tendency not to take into account the consequences of actions), or low self-control (defined as a tendency to act in an automatic rather than in a controlled way) [4, 32, 51]. The overuse of mobile phones has also been related to ADHD symptoms in adolescence [52]. The aforementioned impulsivity traits have been similarly related to risky patterns of use that have little or nothing to do with addictive behavior, such as dangerous use (e.g., phoning while driving) [4, 32] or sexting (i.e., exchange of sexually suggestive pictures or messages via the mobile phone) [53, 54]. Impulsivity, along with psychopathic traits, has also been related to antisocial patterns of mobile phone use (defined as a use involving negative outcomes, such as social disapproval of other's suffering) and feeling compelled to use mobile phones in banned areas [4] or the involvement in cyberbullying behaviors [55]. It is worth noting that this pathway, and its related etiology, finds its theoretical roots in Blaszczynski and Nower's [56] pathway model of disordered 
Fig. 1 A pathway model of problematic mobile phone use (PMPU)

\section{PROBLEMATIC MOBILE PHONE USE}

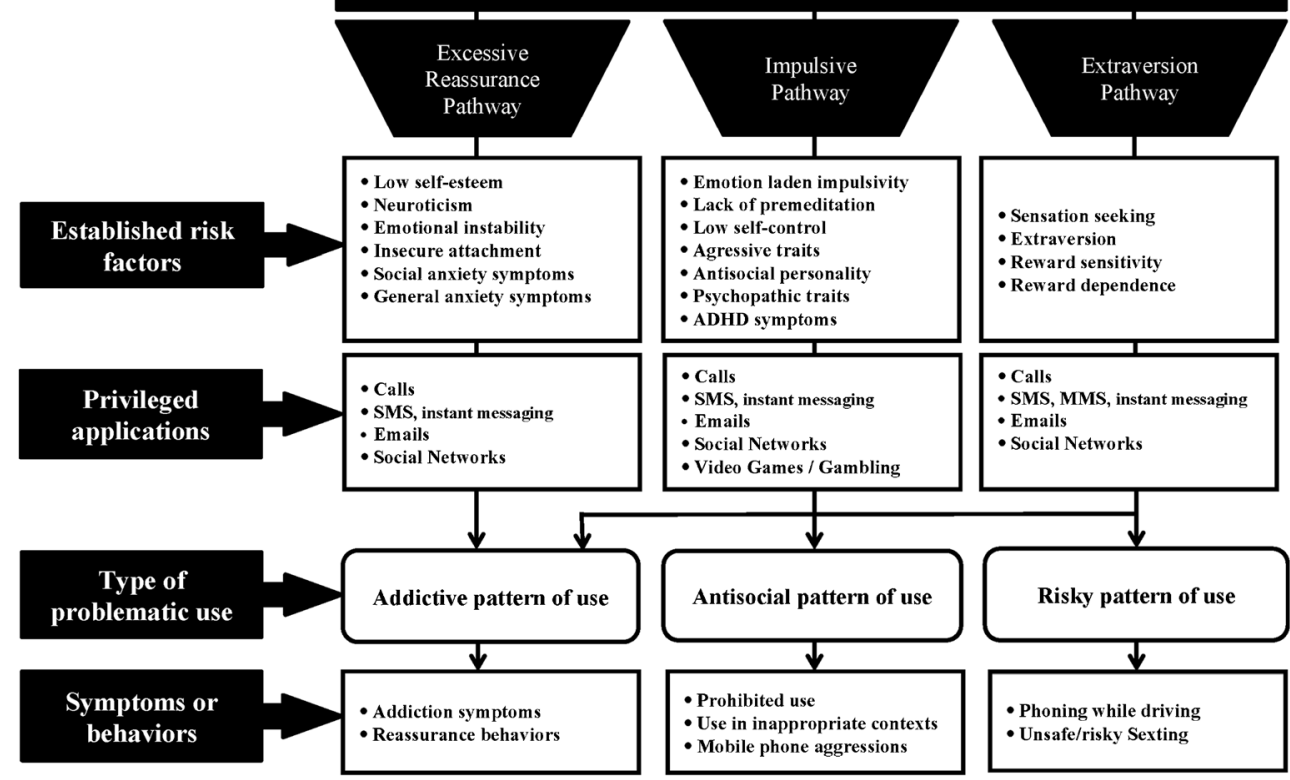

gambling, and that a similar pathway has also been recently related to dysfunctional involvement in online video games [57]. Accordingly, the impulsive pathway is probably the more suited to account for PMPU related to specific activities, such as video gaming or gambling.

The third pathway leading to PMPU is the extraversion pathway. This pathway applies to individuals whose PMPU takes the form of dependence-like symptoms and exaggerated use driven by a strong and constant desire to communicate with others and to establish new relationships. Indeed, several studies have documented that PMPU is associated with high extraversion $[6 \bullet \bullet, 43 \bullet, 46-48]$. Interestingly, a study by Igarashi et al. [47] used structural equation modeling to emphasize that SMS overuse can either result from an extraversion path through which dependency is related to a constant desire to socialize with others, or from a neurotic path involving anxiety about relationship maintenance, which corresponds to the excessive reassurance pathway described above. The extraversion pathway also corresponds to individuals whose PMPU can be related to a constant need for stimulations and a high sensitivity to rewards, and is susceptible to taking the form of either antisocial or risky patterns of use. Indeed, a few studies have linked high sensation seeking to mobile phone-based aggressive behaviors [55], phoning while driving [4], and sexting ${ }^{3}$ [53]. It can therefore be assumed that for individuals with a high level of sensation seeking, such activities promote pleasure and excitement (e.g., sending and/or receiving erotic pictures of themselves or someone else), or help to meet the amount of stimulation

\footnotetext{
${ }_{3}^{3}$ Sexting is not necessarily a problematic activity, although in some cases it can be (e.g., sending erotic pictures to a person that cannot be trusted).
}

they need (e.g., using the mobile phone while driving in a context that requires concentration).

Importantly, these pathways have not to be considered as mutually exclusive, and it is possible that in some cases, PMPU is associated with more than one of the described pathways (e.g., a person characterized simultaneously by poor self-esteem and high impulsivity).

\section{Conclusion}

Two main arguments were presented in the current paper. First, the lack of evidence regarding the validity of the behavioral addiction model applied to PMPU was highlighted. This implied that further research emphasizing potential behavioral and neurobiological similarities and correlates with recognized addictive behaviors is required before PMPU can be considered within the spectrum of behavioral addictions. Second, it was demonstrated that PMPU is a heterogeneous and multi-faceted condition. In support of this proposal, a theoretical model was formulated to guide and stimulate future research in the field. In particular, this model emphasizes on the one hand how apparently similar symptoms of mobile phone addiction (e.g., exaggerated and deregulated use) can be driven by different pathways underlain by distinct psychological processes, and on the other hand how some psychological factors (e.g., impulsivity and extraversion) can lead to various types of PMPU that are not related to addictive use (e.g., antisocial and risky use). To conclude, the study of PMPU as a potential psychiatric disorder is in its infancy, and most 
of the existing research appears to have been undertaken without a sufficient theoretical rationale. Indeed, previous research has adopted a confirmatory approach in which PMPU is considered a priori as a behavioral addiction, rather than an exploratory approach interested in determining its phenomenology and manifestations. Eventually, a prerequisite to define a problematic behavior as a possible psychiatric condition (or public health concern) is that it involves functional impairment as reflected by tangible negative outcomes at the individual, professional, or personal levels $[58,59]$. Regarding PMPU, while dangerous use might obviously lead to severe consequences, such as fatal traffic accidents, if using a mobile phone while driving [60], the outcomes of prohibitive use and addictive use are not necessarily associated with significant functional impairment.

\section{Compliance with Ethics Guidelines}

Conflict of Interest Joël Billieux and Pierre Maurage have received funding from the Belgium National Lottery for research on gambling disorder. Joël Billieux and Olatz Lopez-Fernandez have received funding from the European Commission for Research on the problematic usage of information and communication technology ("Tech Use Disorders"; Grant ID: FP7-PEOPLE-2013-IEF-627999). Pierre Maurage (research associate) is funded by the Belgian National Foundation for Scientific Research (F.R.S.-FNRS, Belgium). Mark Griffiths has received funding for a number of research projects in the area of gambling education for youth, social responsibility in gambling, and gambling treatment from the Responsibility in Gambling Trust, a charitable body who funds its research program based on donations from the gambling industry. Mark Griffiths also undertakes consultancy for various gaming companies in the area of social responsibility in gambling. No conflict of interest exists for Daria Kuss.

Human and Animal Rights and Informed Consent This article does not contain any studies with human or animal subjects performed by any of the authors.

\section{References}

Papers of particular interest, published recently, have been highlighted as:

- Of importance

-• Of major importance

1. Geser H. Toward a sociological theory of the mobile phone. Soziologisches Institut der Univ. Zürich: Online Publications. 2004. Retrieved November 28, 2014, from : http://socio.ch/ mobile/index_mobile.htm. 2004.

2. Blake H. Innovation in practice: mobile phone technology in patient care. Br J Community Nurs. 2008;13(4):160-5.

3. Fjeldsoe BS, Marshall AL, Miller YD. Behavior change interventions delivered by mobile telephone short-message service. Am J Prev Med. 2009;36(2):165-73.

4. Billieux J, Van der Linden M, Rochat L. The role of impulsivity in actual and problematic use of the mobile phone. Appl Cogn Psychol. 2008;22(9):1195-210.
5. Billieux J, Van der Linden M, d'Acremont M, Ceschi G, Zermatten A. Does impulsivity relate to perceived dependence and actual use of the mobile phone? Appl Cogn Psychol. 2007;21(4):527-37.

6.• Bianchi A, Phillips JG. Psychological predictors of problem mobile phone use. Cyberpsychol Behav. 2005;8(1):39-51. The first empirical study that addressed the psychological factors associated with problematic mobile phone use (self-esteem, extraversion, neuroticism). This study has inspired many studies on risk factors for problematic mobile phone use.

7. Thomée S, Harenstam A, Hagberg M. Mobile phone use and stress, sleep disturbances, and symptoms of depression among young adults-a prospective cohort study. BMC Public Health. 2011;11(1):66.

8. Barkana Y, Zadok D, Morad Y, Avni I. Visual field attention is reduced by concomitant hands-free conversation on a cellular telephone. Am J Ophthalmol. 2004;138(3):347-53.

9. White MP, Eiser JR, Harris PR. Risk perceptions of mobile phone use while driving. Risk Anal. 2004;24(2):323-34.

10. Nickerson RC, Isaac H, Mak B. A multi-national study of attitudes about mobile phone use in social settings. Int $\mathrm{J}$ Mob Comm. 2008;6(5):541-63.

11. Nicol A, Fleming MJ. « i h8 u »: the influence of normative beliefs and hostile response selection in predicting adolescents' mobile phone aggression — a pilot study. J Sch Violence. 2010;9(2):21231.

12. Lopez-Fernandez O, Honrubia-Serrano L, Freixa-Blanxart M, Gibson W. Prevalence of problematic mobile phone use in British adolescents. Cyberpsychol Behav Soc Netw. 2014;17(2):91-8.

13. Smetaniuk P. A preliminary investigation into the prevalence and prediction of problematic cell phone use. J Behav Addict. 2014;3(1):41-53.

14. Ha JH, Chin B, Park DH, Ryu SH, Yu J. Characteristics of excessive cellular phone use in Korean adolescents. Cyberpsychol Behav. 2008;11(6):783-4.

15. Yen CF, Tang TC, Yen JY, Lin HC, Huang CF, Liu SC, et al. Symptoms of problematic cellular phone use, functional impairment and its association with depression among adolescents in Southern Taiwan. J Adolesc. 2009;32(4):863-73.

16. Salehan M, Negahban A. Social networking on smartphones: when mobile phones become addictive. Comput Hum Behav. 2013;29(6): 2632-9.

17. Toda M, Monden K, Kubo K, Morimoto K. Cellular phone dependence tendency of female university students. Jpn J Hyg. 2004;59(4):383-6.

18. Carbonell X, Guardiola E, Beranuy M, Bellés A. A bibliometric analysis of the scientific literature on Internet, video games, and cell phone addiction. J Med Libr Assoc. 2009;97(2):102-7.

19. Kwon M, Lee J-Y, Won W-Y, Park J-W, Min J-A, Hahn C, et al. Development and validation of a smartphone addiction scale (SAS). PLoS ONE. 2013;8(2):e56936.

20. King ALS, Valença AM, Silva ACO, Baczynski T, Carvalho MR, Nardi AE. Nomophobia: dependency on virtual environments or social phobia? Comput Hum Behav. 2013;29(1):140-4.

21.• Billieux J. Problematic use of the mobile phone: a literature review and a pathways model. Curr Psychiatr Rev. 2012;8(4):299-307.

The first comprehensive review on problematic mobile phone use published. First, it describes existing tools to assess problematic mobile phone use, and provides assessment guidelines for future studies. Second, a detailed description of established psychosocial risk factors is provided. Finally, a theoretical framework for future research is proposed.

22. Griffiths MD. A 'components' model of addiction within a biopsychosocial framework. J Subst Use. 2005;10(4):191-7.

23. Griffiths MD. Technological addictions. Clin Psychol Forum. 1995;76:14-9. 
24. Weinstock J, Rash CJ. Clinical and research implications of gambling disorder in DSM-5. Curr Addict Rep. 2014;1:159-65.

25. Petry NM, Rehbein F, Gentile DA, Lemmens JS, Rumpf H-J, Mössle T, et al. An international consensus for assessing internet gaming disorder using the new DSM-5 approach. Addiction. 2014;109(9):1399-406.

26. Groman SM, James AS, Jentsch JD. Poor response inhibition: at the nexus between substance abuse and attention deficit/hyperactivity disorder. Neurosci Biobehav Rev. 2009;33(5):690-8.

27. Weafer J, Mitchell SH, de Wit H. Recent translational findings on impulsivity in relation to drug abuse. Curr Addict Rep. 2014;1:289-300.

28. Goudriaan AE, Oosterlaan J, de Beurs E, Van den Brink W. Neurocognitive functions in pathological gambling: a comparison with alcohol dependence, Tourette syndrome and normal controls. Addiction. 2006;101(4):534-47.

29. Billieux J, Lagrange G, Van der Linden M, Lançon C, Adida M, Jeanningros R. Investigation of impulsivity in a sample of treatment-seeking pathological gamblers: a multidimensional perspective. Psychiatry Res. 2012;198(2):291-6.

30. Littel M, Berg I, Luijten M, Rooij AJ, Keemink L, Franken IH. Error processing and response inhibition in excessive computer game players: an event-related potential study. Addict Biol. 2012;17(5):934-47.

31. Dong G, DeVito EE, Du X, Cui Z. Impaired inhibitory control in 'internet addiction disorder': a functional magnetic resonance imaging study. Psychiatry Res. 2012;203(2-3):153-8.

32. Billieux J, Gay P, Rochat L, Van der Linden M. The role of urgency and its underlying psychological mechanisms in problematic behaviours. Behav Res Ther. 2010;48(11):1085-96.

33. Roberts JA, Pirog SF. A preliminary investigation of materialism and impulsiveness as predictors of technological addictions among young adults. J Behav Addict. 2013;2(1):56-62.

34. Billieux J, Philippot P, Schmid C, Maurage P, De Mol J, Van der Linden $\mathrm{M}$. Is dysfunctional use of the mobile phone a behavioural addiction? Confronting symptom-based versus process-based approaches. Clin Psychol Psychother. 2015; in press.

35. Van Holst RJ, van den Brink W, Veltman DJ, Goudriaan AE. Why gamblers fail to win: a review of cognitive and neuroimaging findings in pathological gambling. Neurosci Biobehav Rev. 2010;34(1):87-107.

36. Zhou Z-H, Yuan G-Z, Yao J-J, Li C, Cheng Z-H. An event-related potential investigation of deficient inhibitory control in individuals with pathological Internet use. Acta Neuropsychiat. 2010;22(5): 228-36.

37. Chóliz M. Mobile phone addiction: a point of issue. Addiction. 2010;105(2):373-4

38. Mok J-Y, Choi S-W, Kim D-J, Choi J-S, Lee J, Ahn H, et al. Latent class analysis on internet and smartphone addiction in college students. Neuropsychiatr Dis Treat. 2014;10:817-28.

39. Kim D, Lee Y, Lee J, Nam JK, Chung Y. Development of Korean smartphone addiction proneness scale for youth. PLoS ONE. 2014;9(5):e97920

40. Lin Y-H, Chang L-R, Lee Y-H, Tseng H-W, Kuo TB, Chen S-H. Development and validation of the smartphone addiction inventory (SPAI). PLoS ONE. 2014;9(6):e98312.

41. King ALS, Valença AM, Nardi AE. Nomophobia: the mobile phone in panic disorder with agoraphobia: reducing phobias or worsening of dependence? Cogn Behav Neurol. 2010;23(1):52-4.

42. Lu X, Watanabe J, Liu Q, Uji M, Shono M, Kitamura T. Internet and mobile phone text-messaging dependency: factor structure and correlation with dysphoric mood among Japanese adults. Comput Hum Behav. 2011;27(5):1702-9.

43. Lu X, Katoh T, Chen Z, Nagata T, Kitamura T. Text messaging: are dependency and excessive use discretely different for Japanese university students? Psychiatry Res. 2014;216(2):255-62. This research aims to distinguish excessive versus dependent use of mobile phones. The study comprises two measurement times (5-month interval), and is thus the first longitudinal study in the field of mobile phone problematic use research.

44. Lee Y-K, Chang C-T, Lin Y, Cheng Z-H. The dark side of smartphone usage: psychological traits, compulsive behavior and technostress. Comput Hum Behav. 2014;31:373-83.

45. Lepp A, Barkley JE, Karpinski AC. The relationship between cell phone use, academic performance, anxiety, and Satisfaction with Life in college students. Comput Hum Behav. 2014;31:343-50.

46. Ehrenberg A, Juckes S, White KM, Walsh SP. Personality and selfesteem as predictors of young people's technology use. Cyberpsychol Behav. 2008;11(6):739-41.

47. Igarashi T, Motoyoshi T, Takai J, Yoshida T. No mobile, no life: self-perception and text-message dependency among Japanese high school students. Comput Hum Behav. 2008;24(5):2311-24.

48. Butt S, Phillips JG. Personality and self-reported mobile phone use. Comput Hum Behav. 2008;24(2):346-60.

49. Augner C, Hacker GW. Associations between problematic mobile phone use and psychological parameters in young adults. Int J Public Health. 2012;57(2):437-41.

50. Leung L. Linking psychological attributes to addiction and improper use of the mobile phone among adolescents in Hong Kong. J Child Media. 2008;2(2):93-113.

51. Khang H, Kim JK, Kim Y. Self-traits and motivations as antecedents of digital media flow and addiction: the internet, mobile phones, and video games. Comput Hum Behav. 2013;29(6): 2416-24.

52. Zheng F, Gao P, He M, Li M, Wang C, Zeng Q, et al. Association between mobile phone use and inattention in 7102 Chinese adolescents: a population-based cross-sectional study. BMC Public Health. 2014;14(1):1022.

53. Dir AL, Cyders MA. Risks, risk factors, and outcomes associated with phone and internet sexting among university students in the United States. Arch Sex Behav. 2015;in press.

54. Dir AL, Cyders MA, Coskunpinar A. From the bar to the bed via mobile phone: a first test of the role of problematic alcohol use, sexting, and impulsivity-related traits in sexual hookups. Comput Hum Behav. 2013;29(4):1664-70.

55. Kokkinos CM, Antoniadou N, Markos A. Cyber-bullying: an investigation of the psychological profile of university student participants. J Appl Dev Psychol. 2014;35(3):204-14.

56. Blaszczynski A, Nower L. A pathways model of problem and pathological gambling. Addiction. 2002;97(5):487-99.

57. Billieux J, Thorens G, Khazaal Y, Zullino D, Achab S, Van der Linden M. Problematic involvement in online games: a cluster analytic approach. Comput Hum Behav. 2015;43:28-34.

58. Stein DJ, Phillips KA, Bolton D, Fulford KWM, Sadler JZ, Kendler KS. What is a mental/psychiatric disorder? From DSM-IV to DSMV. Psychol Med. 2010;40(11):1759-65.

59. Billieux J, Schimmenti A, Khazaal Y, Maurage P, Heeren A. Are we overpathologizing everyday life? A tenable blueprint for behavioral addiction research. J Behav Addict. 2015:in press.

60. Violanti JM. Cellular phones and fatal traffic collisions. Accid Anal Prev. 1998;30(4):519-24. 